\title{
Propagation Measurements and Models for Wireless Communications Channels
}

\author{
To achieve ubiquitous PCS, new and novel ways of classifying wireless \\ environments will be needed that are both widely encompassing and \\ reasonably compact.
}

Jørgen Bach Andersen, Theodore S. Rappaport, and Susumu Yoshida

JORGEN BACH ANDER$S E N$ is a professor at Aalborg University and head of the Center for Personkommunikation.

THEODORE S. RAPPAPORT is an associate profes sor of electrical engineering at Virginia Tech.

SUSUMU YOSHIDA is a professor of electrical enginecring at Kyoto University.

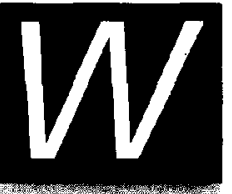

ireless personal communications could in principle use several physical media, ranging from sound to radio to light. Since we want to overcome the limitations of acoustical communications, we shall concentrate on propagation of electromagnetic waves in the frequency range from some hundreds of $\mathrm{MHz}$ to a few $\mathrm{GHz}$. Although there is considerable interest at the moment in millimeter wave communications in indoor environments, they will be mentioned only briefly in this survey of propagation of signals.

It is interesting to observe that propagation results influence personal communications systems in several ways. First there is obviously the distribution of mean powerover a certain area or volume of interest, which is the basic requirement for reliable communications. 'The energy should be sufficient for the link in question, but not too strong, in order not to create cochannel interference at a distance in another cell. Also, since the radio link is highly variable over short distances, not only the mean power is significant; the statistical distribution is also important. This is especially true when the fading distribution is dependent on the bandwidth of the signal. Secondly, even if there is sufficient power available for communications, the quality of the signal may be such that large errors occur anyway. This results from rapid movement through the scattering environment, or impairments due to long echoes leading to inter-symbol-interference. A basic understanding of the channel is important for finding modulation and coding schemes that improve the channel, for designing equalizers or, if this is not possible, for deploying base station antennas in such a way that the detrimental effects are less likely to occur.

In this article we will describe the type of signals that occur in various cnvironments and the modeling of the propagation parameters. Models are essentially of two classes. The first class consists of parametric statistical models that on average describe the phenomenon within a given error. They are simple to use, but relatively coarse. In the last few years a second class of environment-specific mod- els has been introduced. These models are of a more deterministic naturc, characterizing a specific street, building, etc. They are necessarily more time consuming to use, but are also more revealing concerning physical details and hopefully more accurate.

First some key parameters and the measurement of them will be discussed and then the different wireless environments will be treated. The latter topic is divided here into outdoor environments, indoor environments, and radio penetration from outdoor to indoor environments.

\section{The Physics of Propagation}

7 he mechanisms which govern radio propagation are complex and diverse, and they can generally be attributed to three basic propagation mechanisms: reflection, diffraction, and scattering.

Reflection occurs when a propagating electromagnetic wave impinges upon an obstruction with dimensions very large compared to the wavelength of the radio wave. Reflections from the surface of the earth and from buildings produce rcflected waves that may interfere constructively or destructively at a receiver.

Diffraction occurs when the radio path between the transmitter and receiver is obstructed by an impenetrable body. Based on Huygen's principle, secondary waves are formed behind the obstructing body even though there is no line-of-sight (LOS) between the transmitter and receiver. Diffraction explains how radio frequency (RF) energy can travel in urban and rural environments without a LOS path. This phenomenon is also called "shadowing," because the diffracted field can reach a recciver even when it is shadowed by an obstruction.

Scattering occurs when the radio channel contains objects with dimensions that are on the order of the wavelength or lcss of the propagating wave. Scattering, which follows the same physical principles as diffraction, causes energy from a transmitter to be reradiated in many different directions. It has proven to be the most difficult of the three propagation mechanisms to predict in emerging wireless personal communication systems. For example, in urban microccllular systems, lamp posts and street signs 
scatter energy in many directions, thereby providing $\mathrm{RF}$ coverage to locations which might not receive energy via reflection or diffraction. The three mechanisms are illustrated in Fig. 1.

As a mobile radio moves throughout a coverage area, the three propagation mechanisms have an impact on the instantaneous received signal in different ways. For example, if the mobile has a clear LOS path to the base station, diffraction and scattering are not likely to dominate the propagation. Likewise, if the mobile is at street level in a large metropolitan area without a LOS to the base station. diffraction and scattering are most likely to dominate the propagation. As the mobile moves over small distances, the instantaneous received narrowband signal strength will fluctuate rapidly giving rise to small-scale fading. The reason for this is that the field is a sum of many contributions coming from different directions and since the phases are random, the sum behaves as a noise signal, i.e., Rayleigh fading. In small-scale fading, the received signal power may vary by as much as three or four orders of magnitude $(30$ or $40 \mathrm{~dB})$ when the receiver is moved by only a fraction of a wavelength. As the mobile moves away from the transmitter over larger distances, the local average received signal will gradually decrease. Typically, the local average signal is computed over receiver movements of 5 to 40 wavelengths [1-3]. Figure 2 demonstrates the effects of small-scale fading and large scale signal variation for an indoor radio communication system. Notice in the figure that the signal fades rapidly as the receiver moves, but the local average signal changes much more slowly with distance.

In mobile radio systems, communications engineers are generally concerned with two main radio channel issues: link budget and time dispersion. The link budget is determined by the amount of received power that may expected at a particular distance or location from a transmitter, and it determines fundamental quantities such as transmitter power requirements, coverage areas, and battery life. Time dispersion arises due to multipath propagation whereby replicas of the transmitted signal reach the receiver with different propagation delays due to the propagation mechanisms described above. The time-dispersive nature of the channel determines the maximum data rate that may be transmitted without requiring equalization and also determines the accuracy of navigational services such as vehicle location.

\section{Propagation parameters}

Path loss - Link budget calculations require an estimate of the power level so that a signal-to-noise ratio (SNR) or, similarly, a carrier-to-interference (C/I) ratio may be computed. Because mobile radio systems tend to be interference limited (due to other users sharing the same channel) rather than noise limited, the thermal and man-made noise effects are often insignificant compared to the signal levels of cochannel users. Thus, understanding the propagation mechanisms in wireless systems becomes important for not only predicting coverage to a particular mobile user, but also for predicting the interfering signals that userwill experience from other RF sources.

We use path loss (PL) here to denote the local average received signal power relative to the transmit power. This is a useful quantity, since received power is usually measured as a local spatial average rather

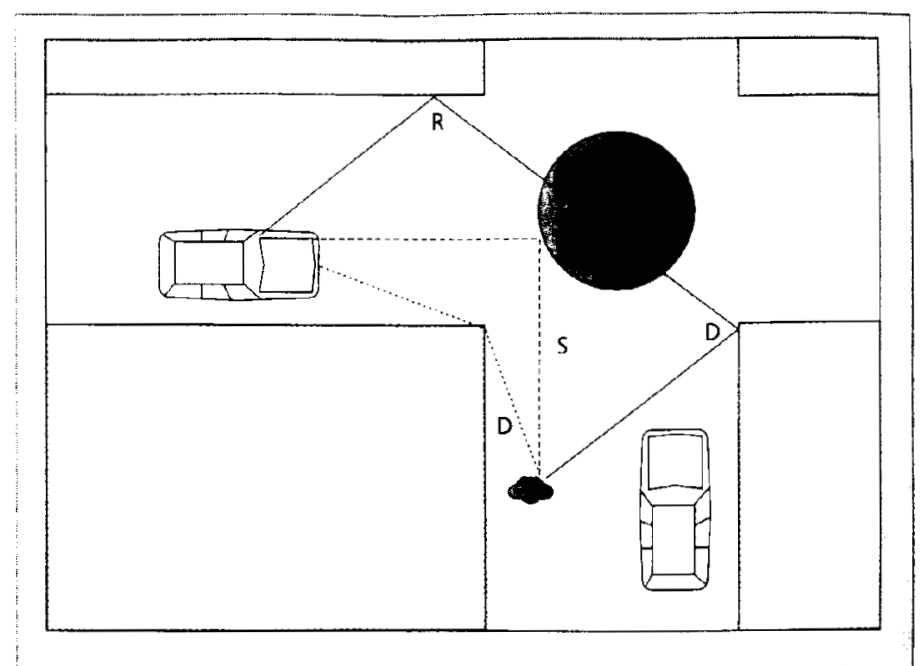

Figure 1. Sketch of three important propagation mechanisms: reflection $(R)$, scattering $(S)$, diffraction $(D)$.

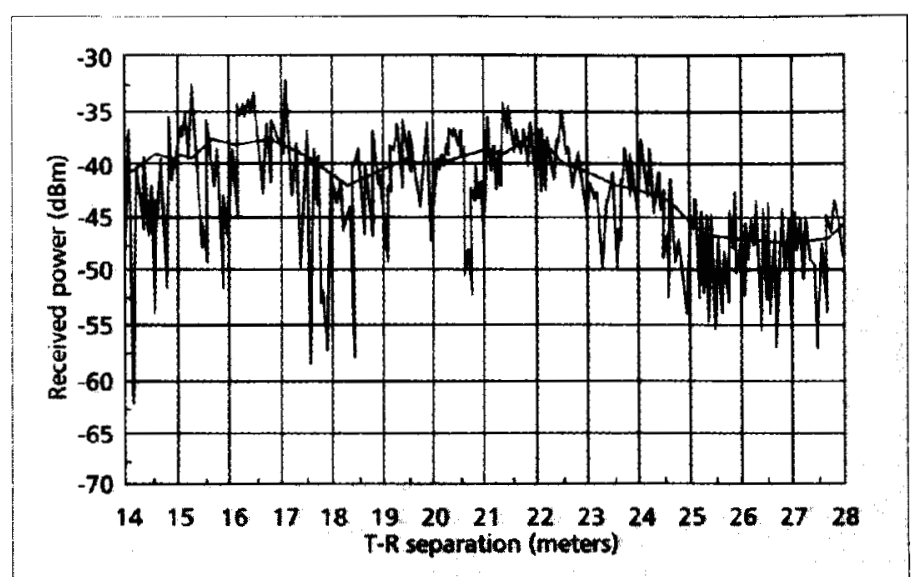

Figure 2. Typical received signal levels for an indoor radio communication system. Notice that small scale fading produces level changes of $20 \mathrm{db}$ or more, where the local average signal level changes much more slowly with distance.

than an instantaneous value. In realistic mobile radio channels, free space does not apply. A general $P L$ model that has been demonstrated through measurements $[1,4]$ uses a parameter, $n$, to denote the power law relationship between distance and received power. As a function of distance, $d, P L$ (in decibels) is expressed as

$$
P L(d)=P L\left(d_{0}\right)+10 n \log \left(d / d_{0}\right)+X_{\sigma}
$$

where $n=2$ for free space, and is generally higher for wireless channels.

The term $P L\left(d_{0}\right)$ simply gives $P L$ at a known close in reference distance $d_{0}$ which is in the far field of the transmitting antenna (typically $1 \mathrm{~km}$ for large urban mobile systems, $100 \mathrm{~m}$ for microcell systems, and $1 \mathrm{~m}$ for indoor systems) and $X_{\sigma}$ denotes a zero mean Gaussian random variable (with units of $d B$ ) that reflects the variation in average received power that naturally occurs when a $P L$ model of this type is used. Since the $P L$ model only accounts for the distance which separates the transmitter 


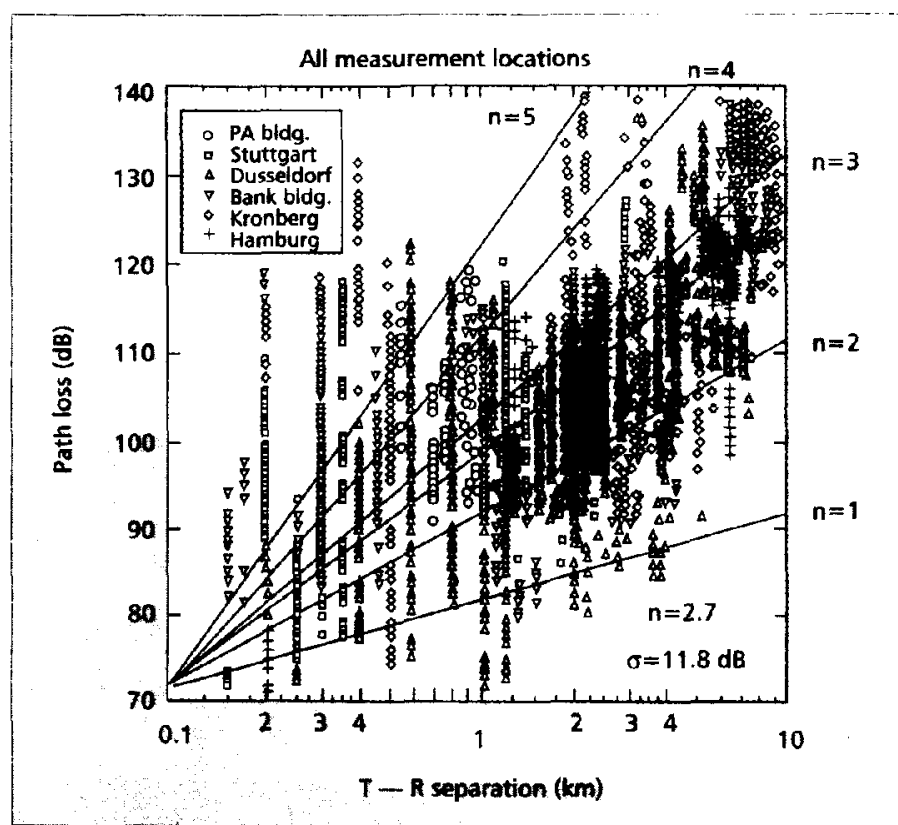

Figure 3. Scatter plot of path loss vs. distance for measurements in five German cities. The path loss is referenced to a free space reference measurement at $d_{0}=100 \mathrm{~m}$. Note that $\sigma$ has a value of $11.8 \mathrm{~dB}$ dure to the large spread of $P L$ values relative to the straight-line fit.

and receiver, and not any of the physical features of the propagation environment, it is natural for several measurements to have the same T-R separation, but to have widely varying $P L$ values. This is due to the fact that shadowing may occur at some locations and not others, etc. The precision of a $P L$ model is thus measured by the standard deviation $\sigma$ of the random variable $X_{\sigma}$, with a smaller value of $\sigma$ reflecting a more accurate $P L$ prediction model.

Figure 3 demonstrates measured values of $P L$ as a function of distance in five cities within Germany and shows the above model superimposed on the measured values. This type of plot is called a scatter plot, and it is useful for quickly assessing the tendencies and variations of path loss throughout a radio system. To achieve lower values of $\sigma$, more site-specific information about the environment is required or smaller coverage distances are used when applying the PL model above [5].

Multipath delay spread - Time dispersion varies widely in a mobile radio channel due to the fact that reflections and scattering occur at seemingly random locations, and the resulting multipath channel response appears random, as well. Because time dispersion is dependent upon the geometric relationships between transmitter, receiver, and the surrounding physical environment, communications engineers often are concerned with statistical models of time dispersion parameters such as the average rms or worst-case values. To demonstrate how rapidly the multipath characteristics may change, consider Fig. 4, which illustrates one of the worst cases of multipath time dispersion ever reported in a U.S. cellular radio system. Figure 4 indicates that multipath energy $18 \mathrm{~dB}$ down from the first arriving signal incurred a $100-\mu$ s excess prop- agation delay. This corresponds to an excess travel distance of over $30 \mathrm{~km}$ ! When the receiver was moved by just a few meters, the observed multipath characteristics became much more tame, with an observed excess delay of no more than $10 \mu$ s. In typical urban cellular systems, the worst case excess delays (at 99 percent probability levels) for echues which are within $10 \mathrm{~dB}$ of the maximum signal are less than $25 \mu \mathrm{s}$ [6].

A collection of power delay profiles, such as is shown in Fig. 4, can be done in many ways. Typically, a channel sounder is used to transmit a wideband signal which is received at many locations within a desired coverage area. This channel sounder may operate in the time or frequency domain. The time domain response will appear as in Fig. 4, and a swept frequency response will reveal that the channel is frequency-selective, causing some portions of the spectrum to have much greater signal levels than others within the measurement band. Of course, the two measurement techniques are equivalent and can be related by the Fourier transform.

Power delay profiles may be averaged over time or over space as the receiver moves about. The averaging interval is key to determining the applicability of the time dispersion statistics, derived from power delay profiles [6-8].

Some important time statistics may be derived from power delay profiles, and can be used to quantify time dispersion in mobile channels. They are: maximum excess delay at $X \mathrm{~dB}$ down from maximum (MED), mean excess delay $(\bar{\tau})$, and rms delay spread $\sigma_{\tau}$. The MED is simply computed by inspecting a power delay profile and noting the value of excess time delay at which the profile monotonically dips below the $\mathrm{X} \mathrm{dB}$ level. For example, in Fig. 4, the MED at $10 \mathrm{~dB}$ down would be $10 \mu \mathrm{s}$. It should be noted that these parameters are also of interest when they are derived from instantaneous impulse responses.

The mean excess delay $(\bar{\tau})$ is the first central moment of the power delay profile and indicates the average excess delay offered by the channel. The rms measure of the spread of power about the value of $\bar{\tau}, \sigma_{\tau}$, is the most commonly used parameter to describe multipath channels. Chuang [9] and more recent simulation studies have confirmed that a good rule-of-thumb is as follows: if a digital signal has a symbol duration which is more than ten times the rms delay spread $\sigma_{\tau}$, then an equalizer is not required for bit error rates better than $10^{-3}$. For such low values of spread, the shape of the delay profile is not important. On the other hand, if values of $\sigma_{\tau}$ approach or exceed $1 / 10$ the duration of a symbol, irreducible errors due the frequency selectivity of the channel will occur, and the shape of the delay profile will be a factor in determining performance.

\section{Outdoor Propagation}

Frequency reuse is obtained using a cellular struc Fure of coverage zones from base stations. In textbooks, one usually sees a honeycomb structure of hexagonally shaped cells. Unfortunately, a real environment changes the propagation conditions such that a cell, defined as the area in which pathloss is at or below a given value, has a much more irregular shape. Furthermore, at isolated places the cells will have pockets inside each other, perhaps due to height 
variations from natural terrain or from man-made structures. Often interference conditions prevent the optimal use of the cellular system because reality does not correspond to the simple mathematical models. Increasing the power to cover dead spots would create other problems. The network planner has some possibilities though, through placing of the base station antenna and to some extent choosing its radiation pattern, to shape the cell extent. Tilting the antenna in a vertical plane is a normal mcasure and by choosing the height above or below rooftops, maybe even down to street level, something can be done to control the size of the cell.

Cells are typically classified roughly according to size as macrocells and microcells. Table 1 presents some distinctive cell features.

\section{Macrocells}

The average path loss is what remains after averaging the path loss over the fast fading due to multipath. The macrocells were the basis for the first generation systems meant for mobile users, and they generally have base stations at high points like broadcasting systems, with a coverage of several kilometers. The key problem is to make some order of the seemingly noise-like path loss existing over an undulating terrain with a variety of land cover, mixed land and sea, etc. The fast Rayleigh fading corresponds to a true noise distribution, but the slow shadowing type variation is remarkably well described by a log-normal distribution, i.e., the average path loss in $\mathrm{dB}$ has a normal distribution. A good reason for this could be that the average path loss is the result of forward scattering over a great many obstacles, each contributing a random multiplicative factor. When converted to $\mathrm{dB}$ this gives a sum of random numbers, which leads to a normal distribution in the central limit.

In the carly days, planning was mainly based on empirical formulas with an experimental background. Okumura [10] did the first comprehensive measurements in 1968 for Japanese environments, and the derived curves have later been transformed into parametric formulas by Hata [4]. It was noted that a good model for the path loss (or the field strength) was a simple power law where the exponent $n$ could vary as a function of frequency, antenna heights etc. Expressed simpiy, on a log-logscale, this is a linear dependency (Eq. 1). The relationship is strictly empirical, but it has proven extremely robust over the years not only in Japanese surroundings, but in other surroundings as well. The reason for this general validity, also for indoor environments, is not completely clear; Walfisch and Bertoni [11] getgood agreement with diffraction over a multiplicity of absorbing screens. There are simple physical situations where the model is exact. One is of course free space corresponding to $n=2$, another is asymptotic propagation over a flat, finitely conducting surface $(n=4)$, and finally, there is forward scattering over a number of absorbing screens of equal height and distance $(n=4)$. The real world in outdoor macrocells leads to $n$ 's between 2 and 4 , with values closer to 4 in urban situations. We shall meet the same model in the indoor case as well.

The models have been refined with a number of correction factors depending on the land cover, different degrees of built-up areas, forests and rural areas, and in some cases supplemented with one or two knife-edge diffractions. Neither Geo-

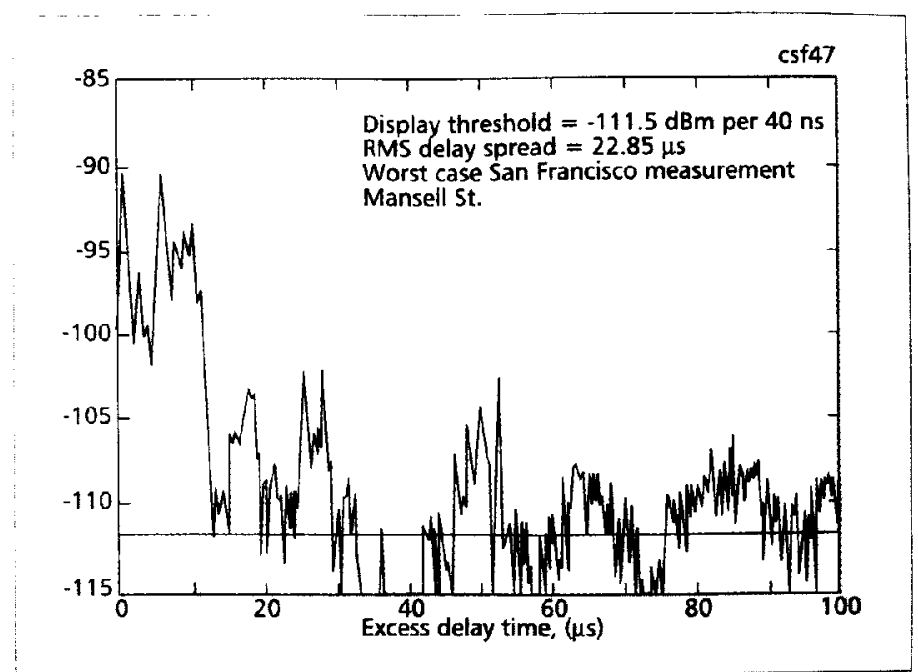

Figure 4. One of the worst cases of multipath time dispersion observed in a cellular radio system. Measurement made inSan Francisco, Califomia.

\begin{tabular}{|l|l|l|}
\hline Item & Macrocell & Microcell \\
\hline Cell radius & 1 to $20 \mathrm{~km}$ & 0.1 to $1 \mathrm{~km}$ \\
\hline Tx power & 1 to $10 \mathrm{~W}$ & 0.1 to $1 \mathrm{~W}$ \\
\hline Fading & Rayleigh & Nakagami-Rice \\
\hline Rms delay spread & 0.1 to $10 \mu \mathrm{s}$ & 10 to $100 \mathrm{~ns}$ \\
\hline Max. bit rate & $0.3 \mathrm{Mb} / \mathrm{s}$ & $1 \mathrm{Mb} / \mathrm{s}$ \\
\hline
\end{tabular}

Table 1. Typical parameters for macro cells and microcells.

metrical Theory of Diffraction (GTD) nor Uniform Theory of Diffraction (UTD) are really successful in macrocells since we are dealing with forward multiple scattering in the transition zones where no good theories exist. The resulting prediction power normally leads to a standard deviation of errors between 6 and $10 \mathrm{~dB}$, which is not really impressive. Even the availability of modern computers and the use of topographical data bases have not resulted in a real break-through in better predictions.

The time domain may also be modeled using geographical data bases, and some success has been achieved in mountainous regions and large cities [12]. In general, the ray tracing routines lack the contributions from random scatterers.

\section{Microcells}

Microcellular Systems - Microcells are attracting much attention simply because they can accommodate more subscribers per unit service area than macrocells. Also, they permit access by low-power portables. Propagation in microcells differs significantly from that in macrocells. As suggested in Table 1, smaller cell coverage with lower antenna height and lower transmission power results in milder propagation characteristics when compared to macrocellular systems. The smaller multipath delay spread and shallow fading imply the feasibility of broadband signal transmission without excessive countermeasure techniques against multipath fading.

Microcells are most frequently planned in an 


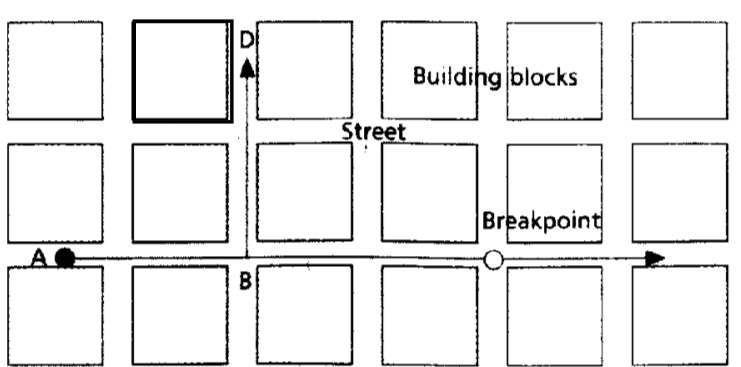

(a)

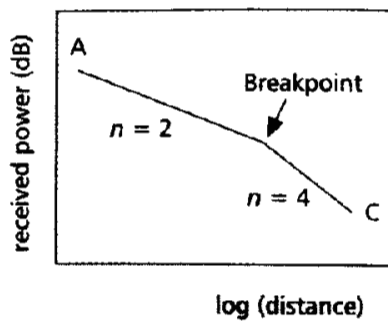

(b)

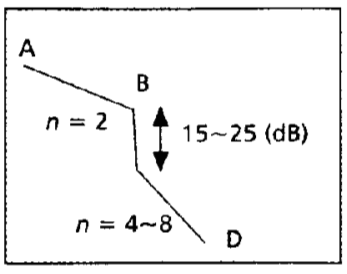

$\log$ (distance)
Figure 5. Ideal urban street layout: a) typical path loss curves along an LOS street b) and along a NLOS street after turning a comer.

urban area where the heaviest tcletraffic is expected. If a transmitter antenna height is lower than the surrounding building height, then most of the signal power propagates along the street. The coverage area extends along the street, thus the name "street microcell" is often used. In suburban areas, microcelis might be used to realize a last quarter mile connection to a wired network. In this case, cell coverage might be circular but strongly affected by the buildings and obstacles.

Street Microcells - A number of studies have been done regarding street microcell propagation where most of the signal power propagates along the street. Let us assume an ideal rectangular street grid with building blocks of relatively uniform height as portrayed in Fig. 5a. In this case, typical path loss curves along the line-of-sight (LOS) path are shown to be characterized by two slopes and a single breakpoint. The path loss exponent is around two, as in free space propagation, from the transmitter up to the breakpoint. Beyond the breakpoint the loss exponent is around four, implying a steeper decrease of the signal strength as illustrated in Fig. $5 \mathrm{~b}$. This breakpoint is approximatcly given by $2 \pi h_{b} h_{m} / \lambda$ where $h_{b}$ is the base antenna height, $h_{m}$ is the mobile antenna height. However, if the receiver turns the corner from the LOS street into a non-lineof-sight (NLOS) street, then the receiver experiences a dramatic decrease of the signal strength by around $20 \mathrm{~dB}$ as shown in Fig. $5 \mathrm{c}$, though this varies depending on the street width and the distance between the transmitter and the corner [13].

On a NLOS street, the signal envelope tends to follow a Rayleigh distribution. On a LOS path, the signal envelope follows not a Rayleigh but a Nakagami-Rice distribution. Multipath delay spread is usually small due to the existence of a dominating, direct signal component.
Modeling and Prediction - Various propaga. tion models for the street microcells based on a ray-optic theory have been proposed. Prediction of microcell coverage based on the ray model is rather accurate compared to the case of macrocells. A fourray model consisting of direct ray, ground-reflected ray. and the two rays reflected by the building walls along the street is often assumed. A six-ray model that takes doubly reflected rays by the building walls or larger-number-of-ray models are reported to give more accurate prediction at the cost of increased computation time [14]. Corner diffraction is another problem attracting much attention. Various models have been investigated starting from a simple knife-edge or wedge diffraction to GTD or UTD with a multiplicity of rays. An analytical path loss model describing microcellular propagation including reflected rays and corner diffraction has been presented in [15].

Due to the irregularity of the building structures, excessive overlap or non-overlapping of the micro cells are sometimes found. Thus, measurementbased methods are also adopted to enhance the accuracy of the prediction [16]. Coverage area is strongly affected by the location of the transmitter antenna. When it is installed in the intersection of the streets in Manhattan, for instance, the waves propagate along the streets in four directions with subsequent corner diffractions at each encountered corner. This generates a coverage area similar to the shape of a diamond. When a transmitter is located on a street between intersec. tions, this makes the cell more elongated in the direction along that street [17]

\section{Indoor Propagation}

ndoor radio communication systems are becom ing increasingly important for extending voice and data communication services within the workplace. Recent trials by major telephone companies show a huge demand and customer acceptance of indoor wireless communications. Some wireless systems interconnect with the PSTN or local PBX to provide a seamless extension of the conventional office telephone system, while other systems offer services separate from the PSTN. Indoor systems can be broken down into three main classes: cordless telephone systems; in-building cellular systems; and local area networks (LANs). Each of these types of emerging communication systems must be designed with the indoor channel in mind.

For indoor communication systems, many design issues such as the distance between servers, expected portable battery life, customer performance expectations, and the appropriate radio link budget are directly linked to the propagation environment. The amount of RF interference that can be expected from cochannel users is an equally important parameter, which is a direct function of the propagation characteristics inside buildings.

\section{Propagation Inside Single-story Buildings}

A great many propagation measurements have been conducted by telecommunication companies, research laboratories, and universities in order to determine reasonable design guidelines and propagation parameters for indoor systems. These measurements show that the particular type of 


\begin{tabular}{|l|l|l|l|}
\hline Building & Freq $(\mathrm{MHz})$ & $n$ & $\sigma \mathrm{dB}$ \\
\hline Retail stores & 914 & 2.2 & 8.7 \\
\hline Groceny store & 914 & 18 & 5.2 \\
\hline Office, hard partition & 1500 & 3.0 & 7.0 \\
\hline Office, soft partition & 900 & 2.4 & 9.6 \\
\hline Office, soft partition & 1900 & 2.6 & 14.1 \\
\hline Factory Los & & & \\
\hline Textile/chemical & 1300 & 2.0 & 3.0 \\
\hline Textilelchemical & 4000 & 21 & 70 \\
\hline Paper/cereals & 1300 & 1.8 & 6.0 \\
\hline Metalworking & 1300 & 16 & 5.8 \\
\hline Suburban home & & & \\
\hline Indoor to street & 900 & 30 & 7.0 \\
\hline Factory OBS & & & \\
\hline Textile/chemical & 4000 & 21 & 9.7 \\
\hline Metalworking & 1300 & 3.3 & 6.8 \\
\hline
\end{tabular}

Table 2. Path loss exponent and standard durawon measured in different buildings.

building has a direct impact on the observed propagation characteristics. This has motivated more recent research that predicts indoor propagation using either statistical or deterministic models.

In order to differentiate propagation phenomena, researchers often classify buildings by the following categorics: rcsidential homes in suburban areas, residential homes in urban areas, traditional older office buildings with fixed walls (hard partitions), open plan buildings with movable wall panels (soft partitions), factory buildings, grocery stores, retail stores, and sports arenas. Hard partitions describe obstructions within the building which cannot be easily moved such as existing walls and aisles. Soft partitions describe movable obstructions such as office furniture panels, which have a height less than the ceiling hcight. Inside a building, propagation geometry may be classified as line of sight (LOS) where the transmitter and receiver are visible to one another or obstructed (OBS), where objects in the channel block a visible propagation path. Often, physical similarities existbetween different types of buildings. For example, factory buildings and grocery stores both contain a large amount of metal inventory and often have few hard partitions within the building. Traditional office buildings, with many walls made of plaster and metal lathe, often have similar propagation characteristics to large residential homes or retails stores that contain many partitions.

As discussed in the section on propagation physics, there are a number of important propagation events that must be measured or modelcd before a reliable wireless system can be designed. Measurements by many researchers have more or less provided similar results, which are summarized below.

Temporal Fading for Fixed and Moving Terminals - For fixed terminals within buildings, measurements have shown that ambicnt motion by people throughout the building causes Ricean fading, with the ratio of specular signal power to multipath signal having a value of about $10 \mathrm{~dB}$. This results in a typical variation of less than $15 \mathrm{~dB}$ for 99.9 percent of the time

In general, a portable receiver moving in a building experiences Rayleigh fading for OBS propagation paths and Ricean fading for LOS paths, regardless of the type of building. The Ricean $\mathrm{K}$ factor can vary from 2 to $10 \mathrm{~dB}$ for portable terminals depending on the structure of the building and the number of multipath components that arrive at the receiver.

Multipath Delay Spread - Buildings that have fewer metal and hard partitions typically have small rms delay spreads, on the order of 30 to $60 \mathrm{~ns}$. Such buildings can support data rates in excess of several $\mathrm{Mb} / \mathrm{s}$ without the need for equalization. However, larger buildings with a great deal of metal and open aisles can have rms delay spreads as largc as $300 \mathrm{~ns}$. Such buildings are limited to data rates of a few hundred kilobits per second without equalization.

Path Loss - Path loss is a measure of the average RF attenuation inside a building, and it is measured by averaging the received signal over several wavelengths at the receiver. Equation (1) describes the path loss situation also for indoor situations where the standard deviation is easily derived from the scatter plot of measured data. $A$ smaller value of $\sigma$ implies that the path loss model provides a better prediction for actual loss within a building. Equation (1) is well suited for system analysis or Monte Carlo simulation when the impact of a large number of users within a building must be considered. Typical values of path-loss exponents and standard deviations for different classes of buildings are given in the literature. Table 2 provides a range of typical values that have been measured in the past.

Molkdar's survey paper on the subject of indoor propagation contains a summary of numerous propagation measurements and models that have been developed [18]. The early works by Devasirvatham [19] and by Salch and Valenzuela [20] have provided a foundation for propagation work in indoor channels.

\section{Propagation Between Floors}

Predicting radio coverage between floors of buildings has proven to be difficult, but measurements have shown that there are general rules-of-thumb that apply, Quantifying this propagation condition is important for in-building wireless systems for multifloored buildings that need to share frequencies within the building. In order to avoid cochannel interference, frequencies must be reused on different floors. The type of building material used between floors has been shown to impact the RF attenuation between floors. Poured concrete over metal lathing is a popular modern construction technique which provides less RF attenuation than does solid steel planks which were used to separate floors in older buildings. The aspect ratio of the building sides also makes a difference. Buildings that have a square footprint have higher attenuation levels between floors than buildings that are rectangular. Also, windows with metallic tint impede RF transmission, thus causing greater
Indoor radio

communica-

tion systems

are becoming

increasingly

important

for extending

voice and

data com-

munication

services

within the

workplace. 
During the

past few

years, indoor

propagation

prediction

techniques

based on ray

tracing have

been used to

reconstruct

the many

possible

reflections

from wall

surfaces

within a

building.

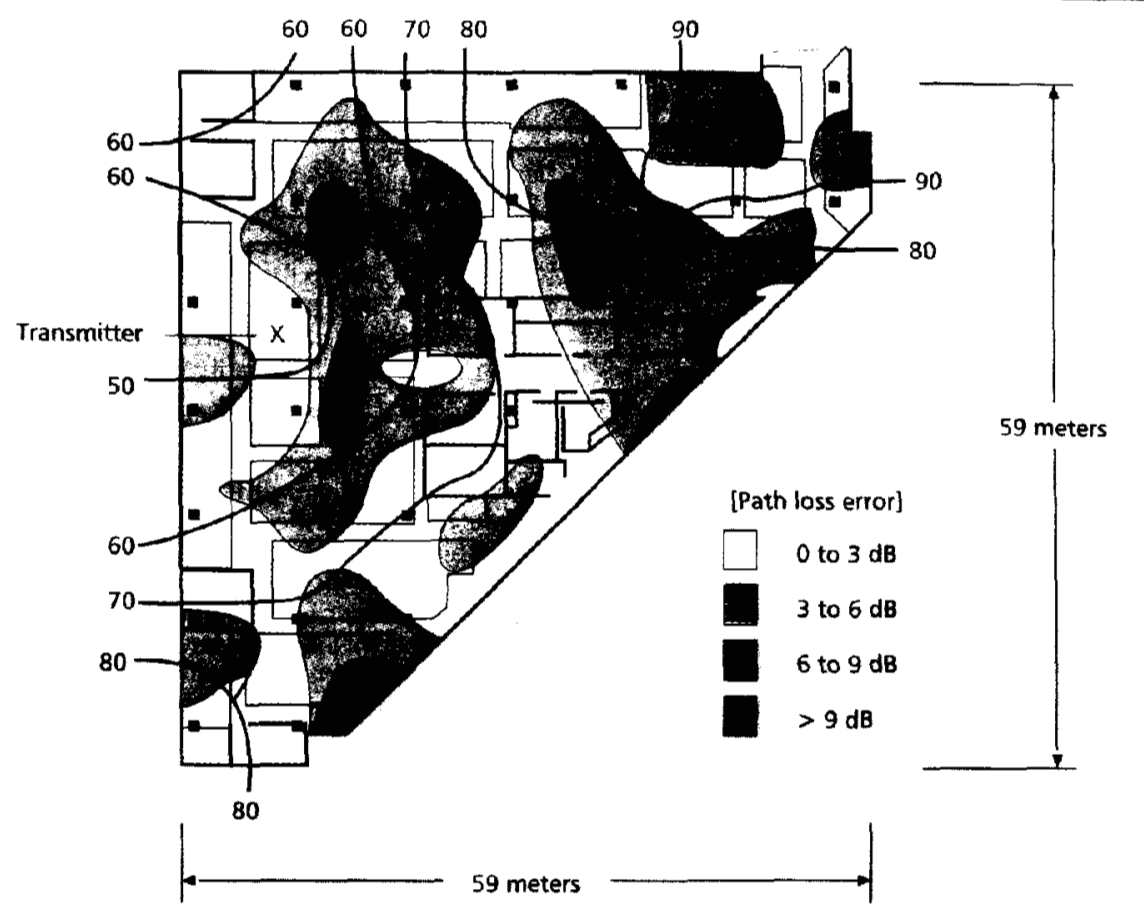

Figure 6. Enror contour which compares measured and predicted path loss using a simple site specific purtition model described in 157 . Note that error is less than $3 \mathrm{~dB}$ over 80 percent of the coverage area.

attenuation between floors of a building.

Measurements have shown that the loss berween floors does not increase linearly in $\mathrm{dB}$ with increasing separation distance. Rather, the greatest floor attenuation factor (FAF) in dB occurs when the transmitter and receiver are separated by a single floor. The overall path loss increases at a smaller rate as the number of floors increase. This phenomenon is thought to be caused by diffraction of radio energy along the sides of a building, as well as scattered energy from neighboring buildings, which can be received on different floors of the same building [21]. Typical values of attenuation between floors is $15 \mathrm{~dB}$ for one floor of separation and an additional 6 to $10 \mathrm{~dB}$ per floor of separation up to four floors of separation. For five or more floors of separation, path loss will increase by only a few $\mathrm{dB}$ for each additional floor.

\section{Computer-aided Design for In-building} Propagation Prediction

Statistical propagation models do not exploit knowledge of the physical surroundings to the degree that building drawings can provide. State-of-the art propagation prediction uses computer-aided design drawings of the indoor environment as a means of representing the physical locations of base stations, receivers, and partitions. For economic reasons, transceivers must be placed strategically so that only desired portions of the building are provided with radio coverage. CAD tools enable a system designer to interactively model the performance of indoor systems. Under this interactive framework, propagation models can be modified to provide a wide range of scenarios and base stations can be added, repositioned, or deleted interactively. Fur- thermore, communication parameters such as received carrier-to-noise thresholds can be adjusted by the user as needed.

Computer-aided design tools, such as AutoCAD, can display both single-floor and multifloor buildings. Building partitions can be identified in AutoCAD and assigned an attribute, so that appropriate propagation models may be applied. Figure 6 shows an example of the modules provided in a typical site modeling tool. Work in $[22,23]$ demonstrated that a simple and surprisingly accurate way to predict RF path loss inside a building is to count partitions between a line drawn between transmitter and receiver. Empirically derived attenuation factors (AFs) may then be applied to each partition which intersects the line. Through an interactive process, parameters such as FAF and AF can be tuned for a particular class of buildings. Measurements made within the environment can then be added to the CAD modeling tool as a means of updating the models in order to provide more accurate measurements at future sites.

During the past $\mathrm{f} \mathrm{ew}$ years, indoor propagation prediction techniques based on ray tracing have been used to reconstruct the many possible reflections from wall surfaces within a building. This approach can easily be accommodated in computer aided design tools and shows promise for reducing the standard deviation $\sigma$ to within $4 \mathrm{~dB}$ over a 50 or $60 \mathrm{~dB}$ dynamic range within buildings [23-25].

\section{RF Penetration into Buildings}

The signal strength received inside of a building due to an external transmitter is important for wireless systems that share frequencies 
with neighboring buildings or with out door systems. Like RF penetration measurements between floors, it is difficult to determine exact models for penetration as only a limited number of experiments have becn published, and they are sometimes difficult to comparc. However, some generalizations can be made from the literature. In measurements reported to date, signal strength received inside a building increases with height. At the lower floors of a building, the urban clutter induces greater attenuation and reduces the level of penetration. At higher floors, a LOS path may exist, thus causing a stronger incident signal at the exterior wall of the building.

RF penetration has been found to be a function of frequency as well as height within the building. Most measurements have considered outdoor transmitters with antenna heights far less than the maximum height of the building under test. Measurcments in Liverpool [26] showed that penetration loss decreases with increasing frequency. Specifically, penetration attenuation values of $16.4,11.6$, and $7.6 \mathrm{~dB}$ were measured on the ground floor of a building al frequencies of $441 \mathrm{MHz}, 896.5$ $\mathrm{MHz}$, and $1400 \mathrm{MHz}$, respectively. Measurements in [27] showed penetration loss of 14.2, 13.4 , and $12.8 \mathrm{~dB}$ for $900 \mathrm{MHz}, 1800 \mathrm{MHz}$, and $2300 \mathrm{MHz}$, respectively. Measurements made in front of windows indicated $6 \mathrm{~dB}$ less penetration loss on average than did measurements made in parts of the building without windows.

Walker [28] measured radio signals into 14 different buildings in Chicago from seven external cellular transmitters. Results showed that building penetration loss decreased at a rate of $1.9 \mathrm{~dB}$ per floor from the ground level up to the 15 th floor and then began increasing above the 15 th floor. The increase in penetration loss at the higher floors was attributed to shadowing effects of adjacent buildings. Similarly, [26] reported penetration loss decreased at a rate of $2 \mathrm{~dB}$ per floor from the ground level up to the 9 th floor and then increased above the 9 th floor. Similar results were also reported in [29].

Mcasurements have shown that the percentage of windows, when compared with the building face surface area, impacts the level of RF penetration loss, as does the presenec of tinted metal in the windows. Metallic tints can provide from 3 to $30 \mathrm{~dB}$ of RF attenuation in a single pane of glass. The angle of incidence of the transmitted wave upon the face of the building also has a strong impact on the penetration loss as shown in [30].

\section{Conclusion}

espite the enormous efforts and progrcss to date, 2 much work remains in the understanding and characterization of wireless communications channels. A trend in 3D numerical modeling is seen, from which time delay statistics as well as coverage and interfercnce might be inferred. This is especially important with the extension to ever-higher daturates. Angle-of-arrival statistics in different environments will also need to be better modeled for usc with adaptive antennas. In general, we may conclude that to achieve ubiquitous PCS new and novel ways of classifying wireless environments will be needed that are both widely encompassing and reasonably compact.

\section{References}

[1] D. Cox, R. Murray, and A. Norris, "800 MHz Attenuation Measured in and Around Suburban Houses," AT\&T Bell Laboratories Tech. $J$., vol. 673, no. 6, July-Aug, 1984, pp. 921-954

[2] W. C. Y. Lee, Mobile Communications Design Fundamentals, Wiley, Second Edition, 1993)

[3] T. 5. Rappaport, "Indoor Radio Communications for Factories of the Future," IEEE Commun. Mag., May 1989, pp. 15-24.

[4] M. Hata, "Empirical formulae for propagation loss in land mobile radio services," iEEE Trans. Veh. Tech., VT-29, 1980, pp. 317-325.

[5] S. Y. Seidel and T. S. Rappaport, “914 MHz Path Loss Prediction Models for Indoor Wireless Communications in Multifloored Buildings," IEEE Trans. Ant. Prop., vol. 40, no. 2, Feb. 1992.

[6] T. S. Rappaport, et. al., "900 MHz Multipath Propagation Measure ments for U.S. Digital Cellular Radiotelephone," IEEE Trans. Veh. Tech. vol. 39, no. 2, May 1990, pp 132-139.

[7] D. C. Cox and R. P. Leck, "Distributions of Multipath Delay Spread and Average Excess Delay for $910 \mathrm{MHz}$ Urban Mobile Radio Paths, IEEE Trans. Ant. Prop., vol. AP 23, March 1975, pp. 206-213

[8] J. Bach Andersen, "Distribution of phase derivatives in mobile communications." IEE Proc., Pt.H, vol. 137, no. 4, Aug. 1990, pp. 197-201

[9] J. C.| Chuang. "The Effects of Time Delay Spread an Portable Radio Communications Channels with Digital Modulation," IEEE JSAC, vol. 5, no. 5, June 1987, pp 879 - 889.

[10] Y. Okumura et. al., "Field strength and its variability in VHF and UHF land mobile radio service, ${ }^{n}$ Rev of the ECL, wol. 16, 1968, pp. 825-873.

[11] I. Walfisch and H. Bertoni, "A Theoretical Model of UHF Propagation in Urban Environments," IEEE Trans. Antennas and Propagation, vol. 36, no. 12, Dec. 1988, pp 1788-1796.

[12] T. Kurner, D. J. Cichon, and W. Wiesbeck. "Concepts and Result for 3D Digital Terrain-Based Wave Propagation Models," IEE ISAC, vol. 11, no. 7, Sept. 1993, pp $1002-1012$

[13] Y. Nagata, et. al., "Measurement and modeling of $2 \mathrm{GHz}$-band out-of-sight radio propagation characteristics under microcellula environments," Proc. PIMRC'91, Sept. 1991, pp. 341-346.

[14] A. J. Rustako, Jr. et. al. "Radio propagation at microwave frequencies for line-of-sight microcellular mobile and personal communications, IEEE Trans Veh. Techn, vol. 40, no. 1, Feb. 1991, pp. 203-210.

[15] d. Wiart, "Micro-cellular Modeling when Base Station Anterna is below Roof TOps," Proc. IEEE Yehicular Technalogy Conference, Jun 1994, Stackholm, pp. 200-204

[16] W. T. Webb, "Sizing up the microcell for mobile radio communications, "Electronics and Commun. Eng. L., pp. 133-140, June 1993.

[17] A. J. Goldsmith and L. J. Greenstein, "A Measurement-Based Model for Predicting Coverage Areas of urban Microcells." IEEE JSAC vol. 11, no. 7. Sept. 1993, pp. 1013-1023

[18] D. Molkdar. "Review on Radio Propagation Into and Within Build ings," IEE Proc vol. 138, no. 1, Feb. 1991

[19] D. Devasirvatham, "Time Delay Spread and Signal Level Measurements of $850 \mathrm{MHz}$ radio waves in building environments," IEEE Tran Ant. Prop., vol. AP-34, no. 11, Nov. 1986, pp. 13001308

[20] A. Saleh and R. Valenzuela, "A statistical model for indoor multipath propagation," IEEE JSAC, vol. SAC 5, no. 2, Feb. 1987, pp. 138-146. 21] W. Honcharenko, H. L. Bertoni, and J. Dailing, "Mechanisms Governing Propagation Between Floors in Buildings," IEEE Trans. Antennas and Propagation, vol. 41, no. 6, June 1993, pp. 787-790.

[22] A. Motley and J. Keenan, "Radio Coverage in Buildings," British Telecom Tech. J., vol. 8, no. 1, Jan. 1990, pp. 19-24.

[23] S. Y. Seidel and T. S. Rappaport, "A Ray Tracing Technique to Pre dirt Path Loss and Delay Spread inside Buildings," IEEE GLOBECOM, DeC. 1992, pp. 1825-1829.

[24] R. Valenzuela. "A Ray Tracing Approach to Predicting Indoor Wireless Transmission," IEEE Veh. Tech. Conf. 1993, pp. 214-218.

[25] C. M. P. Ho, et. al. "Antenna Effects on Indoor Obstructed Wireless Channels and a Deterministic Image-Based Wideband Propagation Model for In-Building Personal Communication Systems, "Int J. Wireless Info. Networks, vol. 1, no. 1, 1994, pp. 61-36.

[26] A. M. D. Turkmani, J. D. Parson, D. G. Lewis, "Radio Propagation Into Buildings at 441,900 , and $1400 \mathrm{MHz}$," Proc 4th Intl. Cont on Land Mobile Radio, Dec. 1987

[27] A. M. D. Turkmani, A. F. Toledo. "Propagation Into and Within Buildings at 900,1800 , and $2300 \mathrm{MHz}$," 1992 IEEE Veh. Tech. Conf.

[28] E. H. Walker, "Penetration of Radio Signals Into Buildings in Cellular Radıo Environments," Bell Sys. Tech. J., vol. 62, no. 9, 1983. [29] J. M. Durante, "Building Penetration Loss at $900 \mathrm{MHz}$," IEEE Veh. Tech.
Conf., 1993 .

[30] J. Horikishi, et al, "1.2 GHz Band Wave Propagation Measurements in Concrete Buildings for Indoor Radio Communications." IEEE
Trans. Veh. Tech. VT-35. no. 4.1986.

\section{Biographies} JORGEN BACH ANOERSEN [F '92] is a professor at Aalborg University.
Aalborg. Denmark and head of Center for Personkommunikation. He is active in international research concerning mobile communications being chairman of COST 231 Working Group on UHF propagation. He is also active in the area of bioelectromagnetics and is presently vicepresident for URSI, the International Scientific Radio Union.

THEODORE S. RAPPAPORT [SM '91] is an associate professor of electrical engineering and founder of the Mobile \& Portable Radio Research Group (MPRG) at Virginia Tech, Blacksburg, Virginia.

SUSUMA YOSHIDA is a professor of electrical engineering at Kyoto University, Kyoto, Japan, leading a research group on wireless personal communications. He is involved in multipath propagation modeling and prediction, sector-antenna diversity, anti-multipath modulatio schemes, and in-service multipath delay spread monitoring, atc.
The

percentage of

windows,

compared

with the

building face

surface area,

impacts the

level of $R F$

penetration

loss, as does

the presence

of tinted

metal in the

windows. 